\title{
Juvenile Paget Disease
}

National Cancer Institute

\section{Source}

National Cancer Institute. Juvenile Paget Disease. NCI Thesaurus. Code C131861.

A bone disorder caused by autosomal recessive mutation(s) of the gene TNFRSF11B, which encodes tumor necrosis factor receptor superfamily member 11B. This condition is characterized by excessive osteoclastic resorption of bone followed by deposition of weak, disorg anized woven bone. Clinical characteristics include short stature, enlarged skull, bony deformities, bone pain, warm skin over the affected bone, joint stiffness, headaches, hearing loss, and elevated serum alkaline phosphatase. 